\title{
Überführung des Dibenzalacetons in Derivate des Diphenylcyclopentans;
}

\author{
von H.J.v. Liebig.
}

[Mitteilung aus dem chemischen Institut der Universität Halle a. S.]

(Eingelaufen am 16. April 1914.)

Durch Einwirkung von konz. Schwefelsäure und Essigsäureanhydrid auf Dibenzalaceton erhielten Vorländer and M. Schrödter ${ }^{1}$ ) die Sulfonsäure eines acetylierten Ketoenols, $\mathrm{C}_{17} \mathrm{H}_{14} \mathrm{O}_{2}$, welches bei der Spaltung mit Alkali in der Hitze Desoxybenzoin neben Acetophenon, Oxalsäure, Benzoesäure und einem Kohlenwasserstoff von der Zusammensetzung des $\alpha$-Methylstilbens ergab.

Dies berechtigte zu der Annahme, daß das Dibenzalaceton bei der Reaktion in ein Derivat des Diphenylcyclopentans übergegangen war:

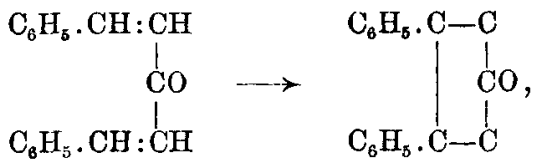

denn nur aus einem solchen können sich durch Spaltung Desoxybenzoin und Methylstilben bilden.

Vorländer und Schrödter wiesen daranf hin, daß das Ketoenol den Produkten nahestehen müßte, welche Japp and seine Mitarbeiter ${ }^{2}$ ) aus Benzil und Aceton dargestellt haben.

Das Anhydracetonbenzil von Japp und das Ketoenol haben die gleiche Zusammensetzung $\mathrm{C}_{17} \mathrm{H}_{14} \mathrm{O}_{2}$, zeigen aber ein gänzlich verschiedenes Verhalten:

Das Ketoenol krystallisiert aus Alkohol in farblosen

1) Ber. d. d. chem. Ges. 36, 1490 (1903); 37, 1133 (1904) von Liebig, Diss. Halle a. S. 1904.

2) Japp a. Miller, Ber. d. d. chem. Ges. 18, 179 (1885). Japp u. Burton, Zentralbl. 1887, 386. Japp u. Lander, ebenda 1896 II, $171 ; 1897$ I, 596; 1901 II, 406. 
v. Liebig, Überführung des Dibenzalacetons usw. 189

Nadeln und schmilzt bei $177^{\circ}$; es gibt eine schwarzviolette Eisenchloridreaktion. Als schwache Säure löst es sich in Alkalilauge anf, doch wenig in Sodalösung. Nach seiner Entstehungsweise ist es aufzufassen als ein Oxydationsprodukt des Dibenzalacetons.

Las Anhydracetonbenzil dagegen ist ein Kondensationsprodukt aus Aceton und Benzil:<smiles>O=C1CC(c2ccccc2)C(O)(c2ccccc2)C1c1ccccc1</smiles>

es krystallisiert in gelben Nadeln; sein Schmelzpunkt liegt bei $147^{\circ}$; es gibt keire Eisenchloridfärbung und ist in ätzendem oder kohlensaurem Alkali nicht löslich.

Auf Veranlassung von Hrn. Prof. Vorländer habe ich versucht, die Konstitution des Ketoenols aufzuklären.

Ich unterwarf es einer oxydativen Spaltung, zuerst durch Behandlung mit Chromsäure in heißer essigsaurer Lösung; später mit Kaliumpermanganat in kalter Acetonlösung: Beide Methoden lieferten neben Kohlensäure Oxalsäure, Benzoesäure, Benzil und Desylessigsäure,

$$
\begin{aligned}
& \mathrm{C}_{6} \mathrm{H}_{5} \cdot \mathrm{CH} . \mathrm{CH}_{2} \mathrm{COOH} \\
& \mathrm{C}_{6} \mathrm{H}_{6} \cdot \mathrm{CO}
\end{aligned}
$$

Die Säure gibt beim Kochen mit Alkalilauge Desoxybenzoin, Acetophenon, Benzoesäure und Oxalsäure.

Auf Grund der oxydativen Spaltung des Enols, der Überführung in Benzil und in Desylessigsäure ist erwiesen, daß bei der eigenartigen Reaktion mit Essigsäureanhydrid und Schwefelsäure tatsächlich die beiden $\beta$-Kohlenstoffe des vibenzalacetons miteinander in Verbindung getreten sind. Sodann darf man folgern, daß im Ketoenol das Methylen der Desylessigsäure enthalten ist:

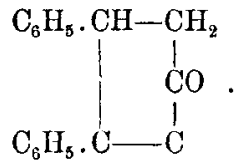


Wenn das Ketoenol ein Derivat des Cyclopentans ist, so mußte es bei der Reduktion mit Jodwasserstoff den bei $47^{\circ}$ schmelzenden Kohlenwasserstoff $\mathrm{C}_{17} \mathrm{H}_{18}$ liefern, welchen zuerst Japp and Burton ${ }^{1}$ ) aus Anhydracetonbenzil mittelst rauchender Jodwasserstofisäure und Phosphor bei $130^{\circ}$ erhielten, das 1,2-Diphenylcyclopentan,

$$
\begin{aligned}
& \mathrm{C}_{8} \mathrm{H}_{5} \cdot \mathrm{CH}-\mathrm{CH}_{2} \\
& \mathrm{C}_{6} \mathrm{H}_{5} \cdot \mathrm{CH}-\mathrm{CH}_{2}
\end{aligned} \mathrm{CH}_{2} .
$$

Es ist mir gelungen, durch Kochen des Enols mit Jodwasserstoffsäure und Phosphor einen Kohlenwasserstoff $\mathrm{C}_{17} \mathrm{H}_{18}$ darzustellen, welcher nach Krystallform, Schmelzpunkt und Zusammensetzung mit dem Jappschen Kohlenwasserstoff vollkommen identisch ist. Zur Kontrolle wurde der Jappsche Körper aus Anhydracetonbenzil dargestellt und mit dem aus dem Ketoenol erhaltenen verglichen.

Die Schlußfolgerungen aus den Oxydations- und Reduktionsprodukten des Enols, die zur Annahme des Cyclopentanringes führten, konnten durch den Einwand, die Ringbildung sei möglicherweise durch die oxydierenden oder reduzierenden Agenzien erfolgt, in Frage gestellt werden. Daß die Verkettung der beiden $\beta$-Kohlenstoffe des Dibenzalacetons nicht sekundär bei Oxydation oder Reduktion des Enols entsteht, sondern im Molekül des Ketoenols an sich vorhanden ist, wurde durch besondere Versuche mit dem Dibenzalaceton festgestellt. Bei der Oxydation des Dibenzalacetons mit übermangansaurem $\mathrm{Kali}$ in Acetonlösung entsteht weder Benzil noch Desylessigsäure, sondern Kohlensäure, Benzaldehyd, Benzoesäure, und bei der Reduktion des Ketons mit Jodwasserstoffsäure und Phosphor bildet sich, nach Versuchen von G. Metge ${ }^{2}$ ), keine Spur des bei $47^{\circ}$ schmelzenden Diphenylcyclopentans.

1) Zentralbl. 1887, 386. Japp und Burton, ebenda 1896 II, 171; Chem. News 73, 253 (1896); Journ. ehem. Soc. 51, 423 (1887); 71, 131 (1897).

2) Diss. Halle a. S. 1904. 
Uas Gesamtverhalten des Ketoenols, seine Eigenschaften als schwache Säure, seine Beziehungen zum Benzil und der Desylessigsäure, sowie zum Anhydracetonbenzil kann man durch folgende Formel zum Ausdruck bringen:<smiles>CCCCCC1CC(c2ccccc2)CC1C(=O)O</smiles>

$(1,2)$-Diphenyleyclopenten-(2)-olon- $(3,4)$ oder<smiles>O=C1CC(c2ccccc2)C(C(=O)O)C1c1ccccc1</smiles>

$(1,2)$-Diphenyleyclo-

pentandion- $(3,4)$

Das Enol-Hydroxyl ist, wie zu erwarten, sehr reaktionsfähig. Man kann es acetylieren, benzoylieren, mit Anilin zu einem Anilid umsetzen, usw. Die Monoacetylverbindung des Enols, die schon Vorländer und Schrödter dargestellt haben, erhielt ich sowohl durch Erbitzen des Enols mit Acetylchlorid, wie auch durch Schütteln der alkalischen Enollösung mit Essigsäureanhydrid. Merkwürdigerweise gewann ich zwei isomere Monobenzoylderivate. Das eine (feinste Nädelchen, hochschmelzend) entsteht aus Enol und Benzoylchlorid bei $100^{\circ}$; das andere (monokline Prismen, tiefschmelzend) erhält man beim Benzoylieren nach Schotten-Baumann. Beide Benzoate lassen sich durch alkoholisches Kali leicht verseifen unter Abspaltung von Benzoesäure, und entsprechen vielleicht den möglichen zwei tautomeren Formen des Enols:
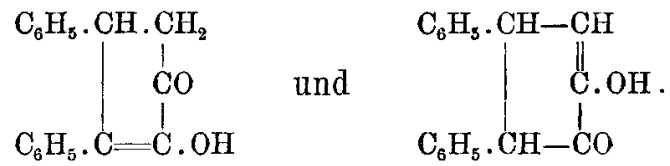

Das in der Strukturformel neben $\mathrm{CO}$ stehende Methylen läßt sich durch Kondensation des Enols mit Benzaldehyd in Gegenwart alkoholisch-wäBriger Kalilauge nachweisen; ich erhielt eine Benzalverbindung,

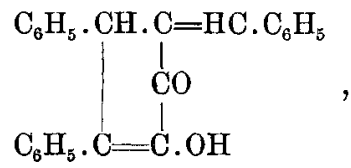


welche, ihrer Struktur entsprechend, stärker sauer ist, als das Enol selbst.

Durch Acetylierung der Benzalverbindung verschwinden die sauren Eigenschaften und die Eisenchloridfärbung:<smiles></smiles>

Als Keton reagiert das Ketoenol mit Hydroxylamin; die nach den gebräuchlichen Oximierungsmethoden dargestellten Substanzen sind amorph und besitzen nicht die Eigenschaften normaler Oxime; ihr stark saurer Charakter weist auf die Bildung einer komplexen Hydroxamsäure hin.

Aus der oben aufgestellten Dionformel muß man entnehmen, daß die Verbindung als Orthodiketon imstande ist, mit Orthodiaminen (Phenylendiamin, Toluylendiamin) Chinoxalinderivate zu bilden. Wider Erwarten konnten jedoch Kondensationsprodukte mit o-Phenylendiamin bisher nicht isoliert werden.

G. Metge hat nachgewiesen, dab das primäre Reaktionsprodukt aus Dibenzalaceton, Essigsäureanhydrid und Schwefelsäure bereits den Cyclopentanring enthält. Der Schwefligsäureverbindung des acetylierten Ketoenols, deren Salze leicht $\mathrm{zu}$ isolieren sind, könnte man folgende Konstitution zuerteilen:<smiles>CO[C@H]1C(c2ccccc2)C(c2ccccc2)CC1(O)O</smiles>

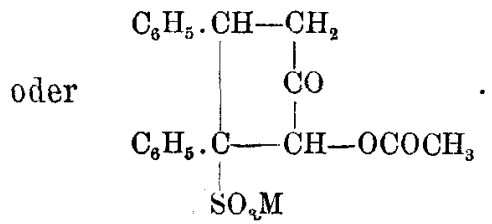

Die Salze liefern bei der Reduktion mit Jodwasserstoff Diphenylcyclopentan, mit Natriumamalgam ein Zwischenprodukt, ein Keton $\mathrm{C}_{17} \mathrm{H}_{16} \mathrm{O}$, wahrscheinlich von der Formel<smiles>O=C1CCC(c2ccccc2)C1c1ccccc1</smiles> 
Das Keton wird auch aus dem Ketoenol bei gemäBigter Reduktion gebildet und ergibt schlieblich mit Jodwasserstoff Diphenylcyclopentan. Die Annahme, daß der Ring bei der Spaltung der Schwefligsäureverbindung durch Alkalien sekundär entsteht, ist durch Gewinnung des gleichen Reduktionsproduktes aus der Schwefligsäureverbindung und aus dem Ketoenol als hinfällig erwiesen.

Gegen die oben stehende Konstitution der acetylierten Schwefligsäureverbindung muß indessen angeführt werden, daß es Metge nicht gelang, die Salze der Verbindung synthetisch durch Einwirkung von sauerem, schwefligsauerem Alkali auf die Acetylverbindung des Ketoenols darzustellen.

Auch umgekehrt, durch Verseifung der Schwefligsäureverbindung mit gelinde wirkenden Mitteln, wie Natriumacetat, Zinkoxyd, Natriumäthylat n. a., war keine Beziehung zum acetylierten Ketoenol aufzufinden, denn beide Gruppen, $\mathrm{COCH}_{3}$ und $\mathrm{SO}_{3} \mathrm{M}$, werden ziemlich gleichzeitig aufgespalten. Las Acetyl hat demzufolge in der acetylierten Schwefligsäureverbindung und im acetylierten Ketoenol eine verschiedene Funktion, vielleicht
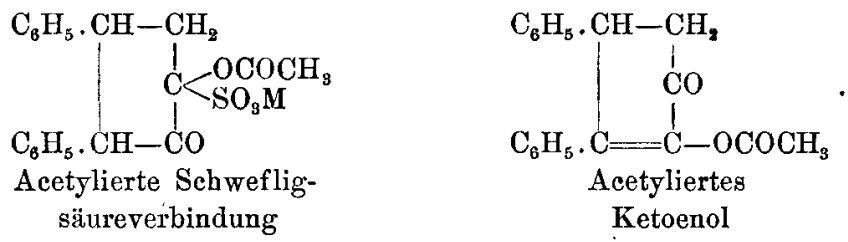

Unaufgeklärt bleibt durch die vorstehende Untersuchung, auf welchem Wege die eigenartige Umwandlung des Dibenzalacetons in das Ketoenol bei der Einwirkung von konz. Schwefelsäure und Essigsäureanhydrid vor sich geht.

Die Verknüpfung der beiden $\beta$-Kohlenstoffatome des Ketons kann entweder die Folge einer direkten Additionsreaktion:
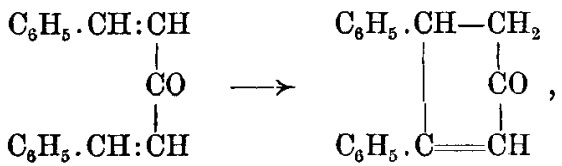

Annalen der Chemie 405. Band. 
oder einer Oxydationsreaktion sein:

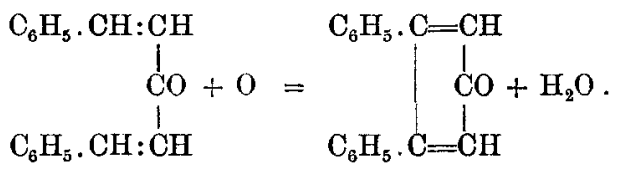

Möglicherweise wirkt auch die Schwefelsäure derart, daß sie sich anfangs an die Doppelbindungen des Dibenzalacetons anlagert und dann in anderer Weise wieder abspaltet, z. B.

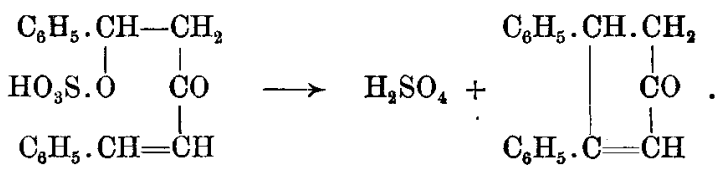

Das kondensierte Keton wird dann $\nabla 0 n$ der Schwefelsäure oxydiert:<smiles>O=C1CC(c2ccccc2)C(C(=O)O)C1c1ccccc1</smiles>

Gegen die direkte Additionsreaktion spricht die Tat sache, daß Dibenzalaceton, in Berührung mit konz. Schwefelsäure, ohne Essigsäureanhydrid, unverändert bleibt. ${ }^{1}$ )

Zugunsten der Oxydationsreaktion ist anzuführen, daß die Verknüpfung zweier Benzalreste zu Benzilderivaten durch Oxydation wiederholt beobachtet worden ist, z. B. der Übergang von Benzalphenylhydrazon in Benzilosazon. ${ }^{2}$ )

Die Abspaltung von Bromwasserstoff ausverschiedenen Brom-Additionsprodukten des Dibenzalacetons führt nach Versuchen von Th. Hell thaler nicht zu einer ringförmigen Verkettung der $\beta$-Kohlenstoffatome des Ketons.

\section{Einwirkung von Essigsäureanhydrid und konzentrierter} Schwefelsäure auf Dibenzalaceton.

$\mathrm{Zu}$ einer Mischung von $50 \mathrm{~g}$ feinzerriebenem reinem Dibenzalaceton und $125 \mathrm{~g}$ Essigsäureanhydrid läßt man

1) Vgl. Ber. d. d. chem. Ges. 36, 1481 und 1484 (1903).

2) Ber. d. d. ehem. Ges. 29, Ref. 863 (1896); 31, 1249 (1898). 
unter fortwährendem Rühren und bei AuBenkühlung mit Eis allmählich $60 \mathrm{~g}$ konz. Schwefelsäure fließen. Das Keton löst sich nach Zusatz der ersten Tropfen Schwefelsäure mit intensiv roter Farbe auf. Man regelt den weiteren Zusatz der Schwefelsäure so, daß sich die Mischung nicht über $25^{\circ}$ erwärmt. Gibt man die Schwefelsäure zu schnell hinzu und erwärmt die Mischung zu stark, so geht die rote Farbe in dunkelbraun mit grüner Fluorescenz über, unter Bildung harziger Zersetzungsprodukte. In der Kälte bildet sich ein mehr oder weniger dicker Krystallbrei der Verbindung aus Dibenzalaceton $+2 \mathrm{H}_{2} \mathrm{SO}_{4}$, welche sich etwa bis zum Verbrauch des ersten Drittels der Schwefelsäure vermehrt, dann aber häufig schon während des Zusatzes des Restes der Säure unter Schwefeldioxydentwicklung zu einer klaren, rotbraunen, sirupartigen Flüssigkeit auflöst. Diese muß in kaltem Wasser klar löslich sein; andernfalls tut man gut, sie in verschlossener Stöpselflasche 2-3 Stunden zu schütteln, wobei sehr bald vollständigeLösung eintritt, welche diefreie acetylierte Schwefligsäure-Verbindung des Diphenylcyclopentenolons enthält:

$$
\left(\mathrm{CH}_{3} \mathrm{CO}\right) \mathrm{C}_{17} \mathrm{H}_{13} \mathrm{O}_{2}\left(\mathrm{H} . \mathrm{SO}_{3} \mathrm{H}\right) \text {. }
$$

Die angewandten Mengen der Reagenzien haben sich erfahrungsgemäß als brauchbar erwiesen; sie entsprechen 1 Mol. Keton, ungefähr 5 Mol. Essigsäureanhydrid und $3 \mathrm{Mol}$. Schwefelsäure. Daß dieser große Überschuß der beiden letzteren im Vergleich zur Menge des Ketons nicht unbedingt zum Zustandekommen der Reaktion erforderlich ist, zeigt ein Versuch, bei dem auf $1 \mathrm{Mol}$. Dibenzalaceton etwas mehr als 1 Mol. Essigsäureanhydrid und kaum 11/2 Mol. Schwefelsäure in Anwendung kamen, und bei dem das gleiche Reaktionsprodukt, allerdings unter starker Verharzung, gebildet wurde.

Auch das mit Essigsäureanhydrid befeuchtete orangefarbene Hydrosulfat des Dibenzalacetons verwandelt sich ohne Zugabe einer weiteren Menge Schwefelsäure nach mehrtägigem Aufbewahren im Exsiccator in die wasser- 
lösliche Schwefligsäureverbindung. Es genügt also schon eine kleine Menge Essigsäureanhydrid zur Herbeiführung der Reaktion.

\section{Kaliumsalz der acetylierten Schwefligsäureverbindung des Diphenylcyclopentenolons, $\left(\mathrm{CH}_{3} \mathrm{CO}\right) \mathrm{C}_{17} \mathrm{H}_{13} \mathrm{O}_{2}\left(\mathrm{H} . \mathrm{SO}_{3} \mathrm{~K}\right)$.}

Zur Darstellung des Kaliumsalzes läßt man die oben gewonnene Lösung aus $50 \mathrm{~g}$ Dibenzalaceton, welche die acetylierte Schwefligsäureverbindung des Diphenylcyclopentenolons enthält, in eine mittelst Salz-Eis-Kältemischung gekühlte Auflösung von $270 \mathrm{~g}$ Kaliumcarbonat in $600 \mathrm{ccm}$ Wasser allmählich unter Rühren einfließen. Das Kaiiumsalz scheidet sich sofort als gelbbrauner Niederschlag aus; es ist schwerer löslich und daher zur Abscheidung besser geeignet, als das von Vorländer und Schrödter näher untersuchte Natriumsalz. Es wird auf großen Porzellannutschen abgesaugt und zur Entfernung des Alkalis mit wenig Wasser gewaschen. Gewicht des an der Luft getrockneten rohen Salzes $80-90 \mathrm{~g}$.

Die Reinheit des Salzes läßt bisweilen zu wünschen übrig; harzige Bestandteile, rote Flocken, gaben Anlab zur Bildung branner schmieriger Niederschläge. Zum Umkrystallisieren wurden $30 \mathrm{~g}$ Kaliumsalz, welches nicht alkalisch reagieren darf, in $400 \mathrm{ccm}$ Wasser unter Erwärmen gelöst und mit Tierkohle gekocht. Aus der gelben filtrierten Lösung scheidet sich das Salz in feinen, farblosen Nadeln als voluminöser Niederschlag ab. Will man das rohe Salz, um Verluste zu vermeiden, nicht auswaschen, so führt man das erste Umkrystallisieren mit essigsäurehaltigem Wasser aus. Das Kaliumsalz enthält 1 Mol. $\mathrm{H}_{2} \mathrm{O}^{1}$ ) Von anderen Salzen, welche G. Metge $e^{2}$ analysiert hat, seien erwähnt:

1) Vgl. Ber. d. d. chem. Ges. 36, 1493 (1903).

$\left.{ }^{2}\right)$ Dissert. Halle a. S. 1904. 
Bariumsalz $\left(\mathrm{C}_{19} \mathrm{H}_{17} \mathrm{O}_{6} \mathrm{~S}\right)_{2} \mathrm{Ba}+4$ bis 5 Mol. $\mathrm{H}_{2} \mathrm{O}$, nach dem Trocknen bei $100-120^{\circ}$.

Calciumsalz $\left(\mathrm{C}_{19} \mathrm{H}_{17} \mathrm{O}_{6} \mathrm{~S}\right)_{2} \mathrm{Ca}+5 \mathrm{Mol} . \mathrm{H}_{2} \mathrm{O}$, farblose Nadeln, in Wasser schwer löslich.

Zinksalz $\left(\mathrm{C}_{19} \mathrm{H}_{17} \mathrm{O}_{6} \mathrm{~S}\right)_{2} \mathrm{Zn}+3 \mathrm{H}_{2} \mathrm{O}$, glänzende Nadeln, aus heißem Wasser gut krystallisierbar.

\section{Darstellung des Retoenols; Diphenylcyclopentenolon,}

$$
\mathrm{C}_{17} \mathrm{H}_{14} \mathrm{O}_{2} \text {. }
$$

Zur Enolbereitung muß das rohe Kaliumsalz ( 80 bis $90 \mathrm{~g}$ ) in Lösung gebracht werden.

Man übergießt es zuerst mit einem Liter heißen Wassers, schlämmt es unter sorgfältigem Zerreiben fein auf, und sänert mit Essigsäure schwach an; man setzt noch 3-4 liter siedendes Wasser hinzu, und kocht unter fortwährendem Rühren auf, so daß eine klare, dunkle Lösung entsteht. Ohne das Sieden zu unterbrechen, gibt man dann allmählich eine Lösung von $75 \mathrm{~g}$ Krystallsoda in $200 \mathrm{ccm}$ Wasser hinzu und kocht einige Minuten: alsbald scheidet sich das Ketoenol in goldbraun schimmernden Schuppen krystallinisch aus; die heiße Flïssigkeit wird dann mit Eisessig angesäuert, wobei Schwefeldioxyd entweicht. Nach dem Erkalten sangt man das Enol ab, wäscht es und trocknet es auf Tontellern. Ausbeute: etwa $32 \mathrm{~g}$ lufttrocknes Enol aus $50 \mathrm{~g}$ Dibenzalaceton, d. i. 60 Proz. der Theorie.

Mitunter scheidet sich nach längerem Stehen noch etwas Enol aus den Mutterlaugen ab. Kaliumsalz aus schlechter Sulfosäure läßt viel Harz beim Aufkochen mit Wasser ungelöst. Giebt man von den Schmieren ab und kocht dann die Lösung mit Soda, so gewinnt man nur wenig unreines Enol.

A us Alkohol, unter Zusatz von Tierkohle, krystallisiert das Ketoenol in farblosen Nadeln. Schmelzp. $176^{\circ}$. Sehr schön krystallisiert es aus Eisessig in langen Nadelbüscheln, und aus Aceton, dem ein wenig Wasser zugesetzt ist, in Spießen, aus Schwefelkohlenstoff in kleinsten Nädelchen. 
Das Enol löst sich leicht in stark verdünnter Kalilauge, weniger in Natronlauge, spurenweise in Sodalösung.

Es reduziert beim Kochen ammoniakalische Silberlösung, aber nicht Fehlingsche Lösung.

Die alkoholische Lösung des Enols wird durch Eisenchlorid dunkelviolett gefärbt.

$\mathrm{Zu}$ allen Versuchen wurde das Enol so, wie es durch Spaltung des Kaliumsalzes gewonnen war, verwendet, ohne weitere Reinigung durch Umkrystallisieren: ein hellgraubraunes Pulver, das auf dem Platinblech restlos verbrennt.

Benzoylverbindungen des Diphenylcyclopentenolons.

Je nach der Art der Benzoylierung bilden sich verschiedene Benzoylderivate:

a) Die Lösung von $1 \mathrm{~g}$ Enol in 8 prozentiger wäßriger Kalilauge wurde bei stetem Überschuß des Alkalis mit 2-3 g Benzoylchlorid unter Abkühlung geschüttelt. Es entstanden schleimige Niederschläge, die sich schwer filtrieren ließen. Das so erhaltene Benzoat (3 $\mathrm{g}$ aus $7 \mathrm{~g}$ Enol) ist nach dem Trocknen äußerst leicht löslich in Alkohol.

Es wurde in wenig warmem Alkohol unter Zusatz von Tierkohle aufgenommen, filtriert, und bei langsamem Verdunsten des Weingeistes zur Krystallisation gebracht: große, farblose Prismen, die an der Wand des Glases in Rosetten anschießen; es schmilzt bei $124^{\circ}$ (Erweichen bei $119^{\circ}$ ).

Die Verbindung ist nicht löslich in Alkalilauge und gibt keine Eisenchloridfärbung.

I. $0,2167 \mathrm{~g}$ gaben $0,6438 \mathrm{CO}_{2}$ und $0,1051 \mathrm{H}_{2} \mathrm{O}$.

II. $0,2041 \mathrm{~g} \quad, \quad 0,6031 \mathrm{CO}_{2} \quad, \quad 0,0964 \mathrm{H}_{2} \mathrm{O}$.

III. $0,2476 \mathrm{~g} \quad, \quad 0,7315 \mathrm{CO}_{\mathrm{z}}, \quad 0,1180 \mathrm{H}_{2} \mathrm{O}$.

\begin{tabular}{cccrr} 
& Ber. für & \multicolumn{3}{c}{ Gef. } \\
& $\mathrm{C}_{24} \mathrm{H}_{13} \mathrm{O}_{3}$ & I & II & III \\
$\mathrm{C}$ & 81,3 & 81,0 & 80,6 & 80,6 \\
$\mathrm{H}$ & 5,1 & 5,4 & 5,3 & 5,3
\end{tabular}


$0,2911 \mathrm{~g}$ gaben, gelöst in $12,19 \mathrm{~g}$ Benzol, eine Gefrierpunktserniedrigung von $0,381^{\circ}$.

$\begin{array}{ll}\text { Ber. für } \mathrm{C}_{24} \mathrm{H}_{18} \mathrm{O}_{3} & 320 \\ \text { Molgew. } \quad 354 & \end{array}$

Verseifung des Benzoats. Die Lösung von etwa $0,5 \mathrm{~g}$ Benzoat in $250 \mathrm{ccm}$ Alkohol, dem einige Kubikzentimeter 50 prozentiger Kalilauge zugesetzt waren, blieb in verschlossener Flasche 14 Tage stehen. Nach dem Abdunsten des Alkohols entsteht ein rotbrauner Niederschlag des in der alkalischen Flüssigkeit schwer löslichen Kaliumsalzes des Enols.

Man löst den Niederschlag in Wasser und fällt mit Essigsäure das Enol aus, welches an seinem Schmelzpunkt und an der Eisenchloridfärbung leicht zu erkennen ist. Benzoesäure ist im alkalischen Filtrat nachzuweisen.

b) Die Mischung von je $1 \mathrm{~g}$ (1 Mol.) Enol und $2 \mathrm{~g}$ (3 Mol.) Benzoylchlorid warde eine Stunde im Wasserbad erhitzt bis zum Verschwinden der Eisenchloridreaktion.

Die harte, braune Masse wurde sodann mit kalter verdünnter Kalilange zur Entfernung der Benzoesäure verrieben. Dem Reaktionsprodukt haftet, auch nach sorgfältigem Auswaschen, etwas braunes Harz an; man entfernt dies mit wenig kaltem Weingeist. Wenn man dann den Körper aus kochendem Weingeist, in dem er schwer löslich ist, unter Zusatz von Tierkohle umkrystallisiert, erhält man die Verbindung in feinsten seidenweichen, weißen Nädelchen; Schmelzp. $173^{\circ}$; unlöslich in Kalilauge; gibt mit Eisenchlorid keine Färbung; erhalten $3 \mathrm{~g}$ Benzoat aus $8 \mathrm{~g}$ Enol.

I. $0,1532 \mathrm{~g}$ gaben $0,4567 \mathrm{CO}_{2}$ und $0,0781 \mathrm{H}_{2} \mathrm{O}$.

II. $0,1057 \mathrm{~g} \quad, \quad 0,3113 \quad \mathrm{CO}_{2} \quad " \quad 0,0546 \mathrm{H}_{2} \mathrm{O}$.

III. $0,1879 \mathrm{~g} \quad \# \quad 0,5592 \quad \mathrm{CO}_{2} \quad \# \quad 0,0907 \quad \mathrm{H}_{2} \mathrm{O}$.

\begin{tabular}{ccccc} 
& Ber. für & \multicolumn{3}{c}{ Gef. } \\
& $\mathrm{C}_{24} \mathrm{H}_{18} \mathrm{O}_{3}$ & I & II & III \\
$\mathrm{C}$ & 81,29 & 81,3 & 80,3 & 81,1 \\
$\mathrm{H}$ & 5,08 & 5,7 & 5,7 & 5,4
\end{tabular}


$0,2527 \mathrm{~g}$ gaben, gelöst in 13,08 Benzol, eine Gefrierpunkts. erniedrigung von $0,297^{\circ}$.

$\begin{array}{rl}\text { Ber. für } \mathrm{C}_{24} \mathrm{H}_{18} \mathrm{O}_{3} & \text { Gef. } \\ \text { Molgew. } \quad 354 & 332\end{array}$

$0,5 \mathrm{~g}$ der hochschmelzenden Verbindung wurden mit alkoholischem Kali ebenso behandelt, wie das isomere tiefschmelzende Benzoat. Nach dem Verdunsten des Alkohols blieb in der stark alkalischen Flüssigkeit ein braunroter, flockiger Niederschlag zurück, aus dessen wäßriger Lösung mit Essigsäure flockiges Enol ausfällt; Schmelzp. $172^{\circ}$, nach dem Umkrystallisieren aus Alkohol.

Das stark alkalische Filtrat enthielt Benzoesäure.

Acetylverbindung des Diphenylcyclopentenolons.

Da das Ketoenol isomere Benzoate liefert, so wurde auch die Acetylierung nach verschiedenen Methoden durchgeführt. Jedoch ergaben sich hier weder bei der direkten Acetylierung durch Frwärmen des Ketoenols mit Essigsäureanhydrid oder Acetylchlorid, noch bei der Acetylierung des in wäßrig-alkalischer Lösung befindlichen Ketoenols isomere Acetylprodukte. Die bereits von Vorländer und Schroedter beschriebene Acetylverbindung schmilzt bei $143-144^{\circ}$.

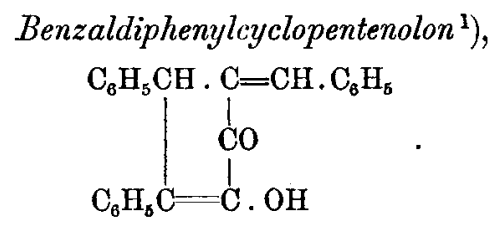

Die Lösung von $1 \mathrm{~g}$ Enol und 2,5 $\mathrm{g}$ Benzaldehyd in verdünntem Weingeist wird mit $1-2 \mathrm{ccm} 10$ prozentiger Kalilauge versetzt und auf dem Wasserbade erwärmt. Die Kondensation ist in wenigen Stunden beendet; man säuert die orange Lösung mit Essigsäure an und verjagt Alkohol and Reste von Benzaldehyd mit Wasser-

1) Vgl. F. W. Gray, Trans. Chem. Soc. 95, 2138 (1909). 
dampf; die ausgefällte Benzalverbindung ist nach dem Trocknen ein hellgelbes, sandiges Pulver. Ausbeute 1,3 g.

Die Verbindung gibt in alkoholischer Lösung eine schwarzbraune Eisenchloridfärbung, ist löslich in ätzendem und kohlensaurem Alkali mit orangeroter Farbe; mit konz. Schwefelsäure entsteht eine scharlachrote Lösung. Sie ist schwer löslich in Chloroforn und Alkohol und krystallisiert daraus in feinen, glänzenden, gelben Nadeln; Schmelzp. 223-224\%

$0,0936 \mathrm{~g}$ gaben $0,2921 \mathrm{CO}_{2}$ und $0,0468 \mathrm{H}_{2} \mathrm{O}$.

$\begin{array}{lcr} & \text { Ber. für } \mathrm{C}_{88} \mathrm{H}_{18} \mathrm{O}_{2} & \text { Gef. } \\ \mathrm{C} & 8 \overline{5}, 20 & 85,11 \\ \mathrm{H} & 5,32 & 5,56\end{array}$

Die Substanz ist schwer verbrennlich; bei einigen Analysen habe ich den Kohlenstoffgehalt um 1 Proz. zu niedrig gefunden.

\section{Acetylderivat der Benzalverbindung.}

Nach dem Kochen mit Essigsäureanhydrid gewinnt man aus der Lösung der Benzalverbindung durch Verdünnen mit Wasser das Acetat als feinen, gelblichen Niederschlag; - unlöslich in Alkalilauge, leicht löslich in Alkohol; es krystallisiert in nahezu farblosen, anscheinend rhombischen Tafeln, Schmelzp. $149^{\circ}$; es gibt keine Eisenchloridfürbung. Es wird durch Kochen mit Alkalilauge sofort verseift, auch tritt die Eisenchloridfärbung des Benzalenols beim Erhitzen der alkoholischen Lösung auf.

Benzoyliert man nach Baumann-Schotten, so erhält man ein nahezu farbloses Benzoylderivat, das sich wie die Acetylverbindung verbält.

\section{Anilid des Diphenylcyclopentenolons.}

Die Lösung von 1 Tl. Enol in 8 Tln. Anilin wurde 8 Stunden auf dem Wasserbad erwärmt; beim Einrühren in verdünnte Salzsäure schied sich ein schmieriger Niederschlag aus, der zu einem dunklen körnigen Pulver eintrocknete; durch wiederholtes Umkrystallisieren aus 
Benzol mit Kohle erhielt ich das Anilid in gelblich weißen, feimen Nädelchen, Schmelzp. $133^{\circ}$, unlöslich in Alkalilauge; es gibt keine Eisenchloridfärbang.

\section{Einwirkung von Phenylhydrazin auf das Enol.}

Läßt man die Lösung von etwa $1 \mathrm{~g}$ Enol und $1 \mathrm{~g}$ Phenylhydrazin in $20 \mathrm{ccm}$ Chloroform einige Tage im verschlossenen Glase stehen, so kann man eine Abscheidung von Wassertropfen beobachten, die auf dem Chloroform schwimmen; aus der dunkelbraunen Lösung wird dann durch Eisessig ein gelbes Hydrazón gefällt. - Vermeidet man einen Überschuß an Chloroform, so scheiden sich mitunter aus der Lösung selbst nach einigen Tagen gelbe Nädelchen aus; durch Konzentrieren gewinnt man eine größere Menge des krystallinischen Hydrazons, das nach dem Auswaschen mit wenig Eisessig bei $175-180^{\circ}$ schmilzt.

\section{Einwirkung von Hydroxylamin auf das Enol.}

Man läßt die Lösung von $1 \mathrm{Mol}$. Enol und 5 Mol. Hydroxylaminhydrochlorid in stark verdünnter Kalilauge einige Tage stehen; dann wird die schwachgefärbte Lösung, der man noch etwas Wasser zugesetzt hat, filtriert und mit Salzsäure unter Kühlung angesäuert; es scheidet sich ein schneeweißer Niederschlag in dichten Flocken aus; er trocknet zu einem hellen, feinen Mehl, Schmelzp. $162^{\circ}$, unter Zersetzung; erhalten: aus $1 \mathrm{~g}$ Enol 0,7-0,8 g. Ich habe den Körper aus keinem der gebräuchlichen Lösungsmittel krystallinisch erhalten können.

$0,0942 \mathrm{~g}$ gaben $7,0 \mathrm{ccm}$ Stickgas bei $10^{\circ}$ u. $748,5 \mathrm{~mm}$ Druck.

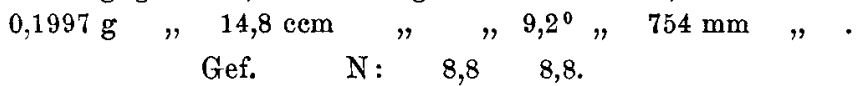

Jie Zahlen deuten auf eine Verbindung mit 2 Mol. Hydroxylamin hin, doch ist die Zusammensetzung nicht genau ermittelt worden, da die Einheitlichkeit der Substanz fraglich war. 
Die Verbindung löst sich außerordentlich leicht in Sodalösung auf, reduziert Fehlingsche Lösung nicht, sondern gibt damit ein graugrünes Kupfersalz, das selbst beim Kochen nicht verändert wird; in Säuren ist sie nicht, oder schwer löslich; mit Eisenchlorid in alkoholischer Lösung entsteht eine tiefgrüne Färbung, die nach einiger Zeit in dunkelbraun übergeht.

Versuche mit o-Phenylendiamin führten zu keinem Kondensationsprodukt. Wenn man molekulare Mengen Enol and Diamin in essigsaurer ${ }^{1}$ ) oder alkoholischer Lösung im Wasserbad erwärmt oder kocht, und die Reaktionsprodukte dann untersucht, so gewinnt man aus den braunen Gebilden das Ketoenol wieder zurück, das durch Krystallform, Schmelzpunkt, Alkalilöslichkeit und Eisenchloridreaktion erkennbar ist.

\section{Oxydation des Diphenylcyclopentenolons mit Permanganat in Acetonlösung.}

Die Lösung von $6 \mathrm{~g}$ Enol in 1 Liter reinen Acetons, das durch Kochen über Permanganat von oxydablen Substanzen befreit ist ${ }^{2}$ ), wird unter Eiskühlung allmählich mit etwa $7 \mathrm{~g}$ staubfeinem Permanganat versetzt; das Permanganat löst sich leicht auf; anfangs verschwindet die rotviolette Tönung rasch, später langsamer.

Wenn die Färbung nach einer halben Stunde noch besteht, ist die Oxydation beendet, dieser Punkt ist nach etwa 10 Stunden erreicht.

Man filtriert dann vom Manganhyperoxyd ab und wäscht mit Aceton nach; der Manganschlamm, der die sauren Oxydationsprodukte in Form ihrer Kaliumsalze enthält, wird mit Wasser ausgelaugt; beim Ansäuern der Laugen mit Salzsäure erhält man ein Gemisch der Rohsäuren (hauptsächlich Benzoesäure), die mit Äther aus-

1) Hinsberg, diese Annalen 237, 327 (1887)

2) F. Sachs, Ber. d. d. chem. Ges. 34, 501 (1901). 
gezogen oder auch abfiltriert werden können. Die Acetonlösung destilliert man ab; der Rückstand, Benzil, ist mehr oder weniger verharzt. Ausbeute aus $30 \mathrm{~g} \mathrm{Enol}$ $8 \mathrm{~g}$ Süuregemisch, $5 \mathrm{~g}$ rohes Benzil.

Gewinnung von Desylessigsäure aus dem Säuregemisch.

Die Säuren werden so lange mit Wasser ausgekocht, bis die Auszüge beim Erkalten sich nicht mehr trüben. Auf diese Weise wird die Benzoesäure entfernt.

Die braunen, harzigen Körner, die zurückbleiben, sind in acetonhaltigem Wasser leicht löslich. Kocht man die Lösung, um das Aceton zu verdampfen, bis zum Auftreten einer feinen Trübung und läßt ganz allmählich erkalten, so krystallisieren schön ausgebildete Doppelpyramiden von Desylessigsäure aus. Frhalten $4 \mathrm{~g}$ aus $8 \mathrm{~g}$ Säuregemisch stark: lichtbrechende Krystalle, die Oktaedern in hohem Grade gleichen. Schmelzp. 158 bis $160^{\circ}$, nach dem Umkrystallisieren aus Alkohol 162-163\%.

$0,2623 \mathrm{~g}$ gaben $0,7237 \mathrm{CO}_{2}$ und $0,1389 \mathrm{H}_{2} \mathrm{O}$.

$0,2370 \mathrm{~g} \quad \# \quad 0,6548 \mathrm{CO}_{2} \quad " \quad 0,1248 \mathrm{H}_{2} \mathrm{O}$.

$0,2195 \mathrm{~g} \quad \# \quad 0,6061 \mathrm{CO}_{3} \quad, \quad 0,1180 \mathrm{H}_{2} \mathrm{O}$.

Ber. für $\mathrm{C}_{18} \mathrm{H}_{14} \mathrm{O}_{3}$

Gef.

$\begin{array}{lrrrr}\mathrm{C} & 75,60 & 75,24 & \mathbf{7 5 , 3 6} & \mathbf{7 5 , 3 2} \\ \mathrm{H} & 5,50 & 5,88 & 5,85 & 5,97\end{array}$

$0,1097 \mathrm{~g}$ Säure in wäBriger Acetonlösung erforderten 4,82 $\mathrm{ccm}$ Barytwasser(F.0,1015); 0,0994 g brauchten $4,05 \mathrm{ccm}(\mathrm{F} .0,1015)$. Ber. für $\mathrm{C}_{16} \mathrm{H}_{14} \mathrm{O}_{3}$

Äqu. Gew. 254

Gef. $224 \quad 242$

Das Silbersalz habe ich aus der neutralen Lösung des Bariumsalzes mit Silbernitrat ausgefällt; schneeweißer, flockiger Niederschlag.

$0,1397 \mathrm{~g}$ hinterließen nach dem Glühen $0,0412 \mathrm{Ag}$, d. i. 29,49 Proz. Ag.

Ber. für $\mathrm{C}_{18} \mathrm{H}_{14} \mathrm{O}_{3}$

Äqu. Gew. $\quad 254$

Gef.

259

Natriumsalz. In einem Tropfen Sodalösung löst sich ein Körnchen der Säure rasch auf, beim Reiben mit einem Glasstab entsteht sofort ein Brei perlmatter- 
glänzender, feiner Schuppen des Na-Salzes, das in Wasser ziemlich leicht löslich ist.

Zur Identifizierung wurde die Säure nach J. Thiele ${ }^{1}$ ) in die beiden Lactone verwandelt und mit der aus Natriumdesoxybenzoin und Bromessigester ${ }^{2}$ ) dargestellten Desylessigsäure verglichen.

\section{Gewinnung von Benzil.}

Das Rohbenzil von der Oxydation des Ketoenols mit Permanganat, ein etwas harziges, hellbraunes, nach Acetophenon riechendes Produkt, läßt sich aus Aceton oder aus Alkohol umkrystallisieren; feine, gelbe Nadeln oder Prismen; Schmelzp. 96-97. Das Benzil wurde, um dessen gewiß zu sein, in das bei $232^{\circ}$ schmelzende Osazon, ferner in Benzilsäure (Schmelzp. $150^{\circ}$ ) verwandelt.

Aus stark verharzten Produkten der Oxydation habe ich das Benzil mit Wasserdampf isoliert; merkwürdigerweise ist es dann fast farblos, schmilzt bei 93-94 $4^{\circ}$, gibt das gewöhnliche Osazon. Die Eigenschaft des Benzils, mit Wasserdämpfen, wenn auch nur in geringem Maße, flüchtig zu sein, ist bisher noch nicht beobachtet worden.

\section{Verhalten der Desylessigsäure gegen Kalilauge.}

Vorländer und Schrödter ${ }^{3}$ ) erhielten beim Kochen des Enols $(20 \mathrm{~g})$ mit verdünnter Katilauge Desoxybenzoin (5 g) und Acetophenon (1,2 g); ich habe dieselben Produkte auch aus Desylessigsäure gewonnen, daneben auch Oxalsäure und Benzoesäure. Man kann demnach für die Zersetzung des Ketoenols eine oxydative, hydrolytische Aufspaltung zu Desylessigsäure und eine weitere Zersetzung dieser Säure annehmen.

1) Diese Annalen 306, 196 (1899) und 319, 164 (1901).

2) Knoevenagel, Ber. d. d. chem. Ges. 21, 1350 (1888); J. Thiele, diese Annalen 319, 163 (1901).

s) a. a. $\mathrm{O}$. 
Kochen des Enols mit konz. Kalilauge führt, nach Vorländer und Schrödter ${ }^{1}$ ), zu einem ungesättigten Kohlenwasserstoff $\mathrm{C}_{15} \mathrm{H}_{14}$, dem $\boldsymbol{c}$-Methylstilben. Desylessigsäure wird unter diesen Umständen hauptsächlich in Benzoesäure verwandelt, wie ich beobachtet habe. Doch kann das Methylstilben durch Spaltung des Ketoenols neben Oxalsäure entstehen:

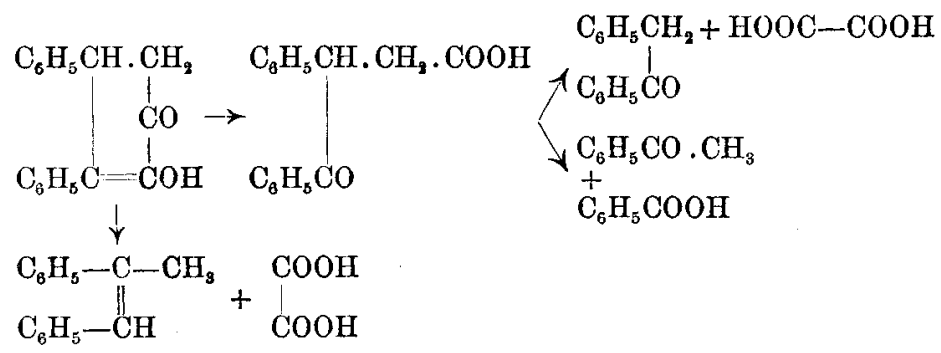

a) $4 \mathrm{~g}$ Desylessigsäure wurden in einer Kupferflasche mit $200 \mathrm{~g} 15$ prozentiger Kalilauge $6^{1 / 2}$ Stunden am Rïckflußkühler gekocht. Die nachfolgende Wasserdampfdestillation ergab in $1 \frac{1}{2}$ Liter Destillat $0,46 \mathrm{~g}$ Desoxybenzoin als flockig krystallinischen Niederschlag. Das von dem Keton befreite Uestillat wurde mit essigsaurer Phenylhydrazinlösung geschüttelt; nach 12 stündigem Stehen hatte sich Acetophenonphenylhydrazon in feinen, gelblichen Krystallschuppen abgeschieden, die nach einigen Stunden auf dem Tonteller zu einem braunen Harz zerflossen. Aus der alkalischen Flüssigkeit in der Flasche erhielt ich durch Ansäuern und Ausäthern 1,72 g Benzoesäure; in den ausgeätherten sauren Mutterlaugen wurde nach Zugabe von Natriumacetat die Oxalsäure als Kalksalz gefällt; $0,22 \mathrm{~g} \mathrm{CaO}$ zeigten an: $0,35 \mathrm{~g}$ Oxalsäure.

Im ganzen gewonnen aus $4 \mathrm{~g}$ Desylessigsäure: $1,72 \mathrm{~g}$ Benzoesäure, $0,35 \mathrm{~g}$ Oxalsäure, $0,46 \mathrm{~g}$ Desoxybenzoin, neben einer nicht gewogenen Menge Acetophenon.

b) $2 \mathrm{~g}$ Desylessigsäure wurden in der Kupferflasche mit $125 \mathrm{~g} 49$ prozentiger Kalilauge gekocht und gleich- 
zeitig mit Wasserdämpfen destilliert. In den ersten $200 \mathrm{ccm}$ Destillat waren nur Spuren von Desoxybenzoin enthalten; weitere $100 \mathrm{ccm}$ Kondenswasser blieben klar; dagegen erhielt ich diesmal $1,89 \mathrm{~g}$ nahezu reine Benzoesäure; berechnet $1,92 \mathrm{~g}$. Oxalsäure konnte kaum nachgewiesen werden, Acetophenongeruch undeutlich. Bei einem anderen Versuch lieferten $2 \mathrm{~g}$ Desylessigsäure und $50 \mathrm{ccm} 50$ prozentiger Kalilauge nach 17 stündigem Erhitzen unter Rückfluß ebenfalls nur ganz wenig Desoxybenzoin neben $0,75 \mathrm{~g}$ Benzoesäure.

Reduktion des Diphenylcyclopentenolons mit Jodwasserstoff; Diphenylcyclopentan, $\mathrm{C}_{17} \mathrm{H}_{18}$.<smiles>c1ccc(C2CCC[C@@H]2c2ccccc2)cc1</smiles>

Ich habe den von F. Japp und seinen Mitarbeitern beschriebenen Kohlenwasserstoff ${ }^{1}$ ) durch Erhitzen des Ketoenols mit rauchender Jodwasserstoffsäure (spez. Gew. 1,96) im Rohr auf $130^{\circ}$ und auch durch Kochen mit konz. Säure (spez. Gew. 1,7), am Rückflußkühler erhalten; die letztere Methode ist weit bequemer als die erstere.

$10 \mathrm{~g}$ Enol und $10 \mathrm{~g}$ roter Phosphor werden in einem Kölbchen mit einem großen Überschuß, etwa $100 \mathrm{ccm}$ konz. Jodwasserstoffsäure (spez. Gew. 1,7) gemischt. Man gibt Bimssteinstückchen hinzu, verbindet das Kölbchen mittelst Asbestpfropfens mit einem Rückflußkühler und erhitzt zum gelinden Sieden über möglichst kleiner Flamme; nach 14 Stunden ist die Reaktion beendet; mitunter ist ein krystallinisches Sublimat im Kühlrohr; in der hellen sirupösen Flüssigkeit ist ein weiches rotes Harz suspendiert, man gießt ab und destilliert das Harz

1) Journ. chem. Soc. 51, 423 (1887); 71, 130 (1897); 79, 1023 (1901); Zentralbl. 1887, 386; 1896 II, 171; 1897 I, 596; 1901 II, 406 u. 579. 
mit Wasserdämpfen. ${ }^{1}$ ) Mit dem Kondenswasser gehen farblose Öltröpfchen über, die in der mit Eis gekühlten Vorlage zu weißen, krystallinischen Flocken erstarren. Die Destillation wird so lange fortgesetzt, bis nichts mehr übergeht. Ausbeute aus $10 \mathrm{~g}$ Enol etwa $6 \mathrm{~g}$ Kohlenwasserstoff, d. i. 68 Proz. der Theorie.

Der Körper ist fast rein, Schmelzp. $45^{\circ}$. Er krystallisiert aus Alkohol in farblosen, langen, flachen Nadeln, Schmelzp. $46^{\circ}$.

Der Kohlenwasserstoff ist mit dem von Japp beschriebenen und nach dessen Verfahren aus Anhydracetonbenzil auch von mir dargestellten Produkte vollkommen identisch.

$0,2783 \mathrm{~g}$ gaben $0,9370 \mathrm{CO}_{2}$ und $0,2154 \mathrm{H}_{2} \mathrm{O}$.

$\begin{array}{ccc} & \text { Ber. für. } \mathrm{C}_{17} \mathrm{H}_{18} & \text { Gef. } \\ \mathrm{C} & 91,87 & 91,81 \\ \mathrm{H} & 8,13 & 8,60\end{array}$

$0,3602 \mathrm{~g}$ gaben in $16,6 \mathrm{Benzol} 0,515^{\circ}$ Gefrierpunktserniedrigung. $\begin{array}{lll}\text { Ber. für } \mathrm{C}_{17} \mathrm{H}_{18} & \text { Gef. } \\ \text { Molgew. } & 222 & 215\end{array}$

Gelegentlich einiger Versuche mit dem Kohlenwasserstoff $\mathrm{C}_{17} \mathrm{H}_{18}$ erhielt ich eine aus Eisessig in feinen, rhombischen Blättchen krystallisierende Tetranitroverbindung, Schmelzp. $217^{\circ}$ unter Zersetzung.

Man trägt den feinzerriebenen Kohlenwasserstoff unter Rühren allmählich in eiskalte rauchende Salpetersäure (spez. Gew. 1,5) ein, worin er sich auflöst. Beim Eingießen in viel Wasser wird die Verbindung in weißen Flocken ausgefällt. Ausbeute theoretisch.

Aus Salpetersäure krystallisiert der Körper in langen Nadeln.

$0,1190 \mathrm{~g}$ gaben $13,75 \mathrm{ccm}$ feuchtes Stickgas bei $10,5^{\circ}$ und $769 \mathrm{~mm}$ Druck.

1) Die Wasserdampfdestillation ist viel weniger umständlich, als die Vakuumdestillation, welche Japp angibt. 
$0,1533 \mathrm{~g}$ gaben $17,70 \mathrm{ccm}$ feuchtes Stickgas bei $10,3^{\circ}$ und $76 \overline{5}, 5 \mathrm{~mm}$ Druck.

$\mathrm{N} \quad \begin{array}{cc}\text { Ber. für } \mathrm{C}_{17} \mathrm{H}_{14}\left(\mathrm{NO}_{2}\right)_{4} & \text { Gef. } \\ 13,93 & 13,97 \quad 13,91\end{array}$

Reduktion der acetylierten Schwefligsäureverbindung des

Dipherylcyclopentenolons mit Joduasserstoff;

nach Versuchen von G. Metge. ${ }^{1}$ )

Man erhitzt eine Mischung von $5 \mathrm{~g}$ Kaliumsalz mit ó g rotem Phosphor und $20 \mathrm{ccm}$ rauchender Jodwasserstoffsäure am Rückflußkühler zum Sieden. Die Abspaltung und Reduktion der schwefligen Säure macht sich bald durch Entweichen von Schwefelwasserstoffgas bemerkbar. Nach 4-5 stündigem Sieden läßt man die Mischung erkalten, befreit das aus rotem Phosphor und organischen Substanzen bestehende Gemenge von der überstehenden saueren Flüssigkeit und destilliert es mit Wasser, um das Diphenylcyclopentan mit Wasserdampf überzutreiben. Der Kohlenwasserstoff sammelt sich in farblosen, bald erstarrenden Tropfen in der Vorlage und krystallisiert aus Alkohol in langen, weißen Nadeln; Schmelzp. 47 ${ }^{\circ}$; er ist identisch mit dem von Japp gewonnenen und oben beschriebenen Diphenylcyclopentan.

Reduktion des Kaliumsalzes der acetylierten Schwefligsäureverbindung und des Diphenylcyclopentenolons mit Natriumamalgam;

nach Versuchen von G. Metge.

$30 \mathrm{~g}$ rohes Kaliumsalz wurden in einem Liter Wasser unter Zusatz einiger Tropfen Essigsäure durch Erwärmen gelöst. Nach der Filtration und Abkühlung auf Zimmertemperatur wurde die klare gelbbraune Flüssigkeit in einen hohen Porzellanbecher gegossen und unter stetem Durchleiten eines sehr starken Kohlensäurestromes mit etwa $300 \mathrm{~g} 3$ prozentigem Natriumamalgam, das nach

1) Dissert. Halle a. S. 1904.

Annalen der Chemie 405. Band. 
und nach in bohnengroßen Stücken zugesetzt wurde, reduziert. Unter Bildung von Schwefelwasserstoff vollzog sich die Reaktion in etwa 2 Stunden. Es schied sich ein schmutziggrauer sandiger Körper in wechselnder Menge aus, gewöhnlich etwa $3 \mathrm{~g}$ Rohprodukt. Aus Weingeist krystallisierte er beim raschen Abkühlen in glitzernden, kleinen sechsseitigen Prismen und Doppelpyramiden. Das geeignetste Mittel zum Umkrystallisieren ist Methylalkohol, aus dem der Körper in farblosen, durchsichtigen, harten Krystallen sich ausscheidet. Er ist in Sodalösung und Alkalilauge schwer löslich. Gegen kalte alkalische Kaliumpermanganatlösung ist er beständig. Die alkoholische Lösung wird durch Eisenchlorid nicht gefärbt. Er schmilzt bei $175^{\circ}$. Nach achttägigem Stehen des lufttrocknen Reduktionsproduktes über Schwefelsäure im Vakuumexsiccator zeigte sich kein Gewichtsverlust.

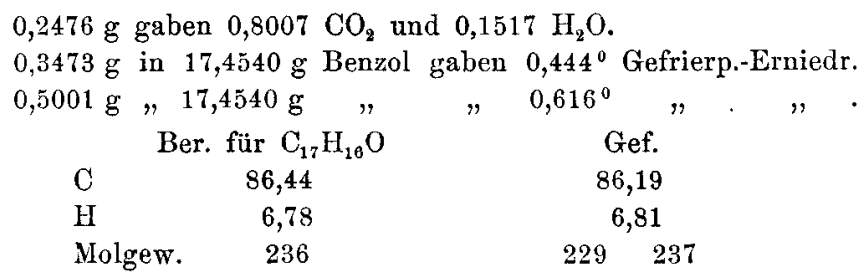

Die gleiche Verbindung $\mathrm{C}_{17} \mathrm{H}_{16} \mathrm{O}$ erhält man, wenn man eine essigsaure Lösung des Salzes mit Natriumamalgam versetzt. Man fügt der Lösung etwas mehr als die dem frei werdenden Alkali äquivalente Menge Eisessig zu and bewirkt die Vermischung mit einem Rührwerk. Man verwendet auf $30 \mathrm{~g}$ Rohsalz etwa $400 \mathrm{~g}$ Amalgam und $31 \mathrm{~g}$ Eisessig. Die Abscheidung erfolgt vielleicht etwas schneller als im Kohlensäurestrom. Die Ausbeute ist bei dieser Methode die gleich geringe wie bei der ersteren. In seinen Eigenschaften unterscheidet sich der Körper nicht ron dem soeben besprochenen.

$0,1220 \mathrm{~g}$ gaben $0,3861 \mathrm{CO}_{2}$ und $0,0801 \mathrm{H}_{2} \mathrm{O}$.

$\begin{array}{ccc} & \text { Ber. für } \mathrm{C}_{\mathbf{1 7}} \mathrm{H}_{\mathbf{1 6}} \mathrm{O} & \text { Gef. } \\ \mathrm{C} & 86, \mathbf{4 4} & 86,31 \\ \mathrm{H} & 6,78 & 7,29\end{array}$


Zur Reduktion des Diphenylcyclopentenolons löst man $8 \mathrm{~g}$ rohes Enol in etwa $1 / 2$ Liter 5 prozentiger Kalilauge. Bei allmählichem Zusatz von Natriumamalgam bei Zimmertemperatur vollzieht sich die Reduktion, allerdings sehr langsam. Die Ausbeute ist auch hier gering. Man gewinnt meist nur etwa $2 \mathrm{~g}$ Rohprodukt durch Ausschütteln mit $\ddot{A}$ ther. Große Mengen harziger, in Äther schwer löslicher Produkte bleiben zurück. Der Körper ist mit dem aus dem Kaliumsalz gewonnenen identisch.

I. $0,1761 \mathrm{~g}$ gaben $0,5612 \mathrm{CO}_{2}$ und $0,1161 \mathrm{H}_{2} \mathrm{O}$.

$0,1669 \mathrm{~g}$ in $10,3135 \mathrm{Benzol}$ gaben $0,361^{\circ}$ Gefrierp.-Erniedr.

II. $0,1869 \mathrm{~g}$ gaben $0,5908 \mathrm{CO}_{2}$ und $0,1209 \mathrm{H}_{2} \mathrm{O}$.

$0,1669 \mathrm{~g}$ in 15,0145 Benzol gaben $0,249^{\circ}$ Gefrierp. Erniedr.

Ber. für

$\mathrm{C}_{17} \mathrm{H}_{16} \mathrm{O}$

$\mathrm{C}$

H

Molgew.
86,44

6,78

236
Gef.

$\begin{array}{cc}\text { I } & \text { II } \\ 86,91 & 86,21 \\ 7,33 & 7,18 \\ 229 & 228\end{array}$

Die bei $175^{\circ}$ schmelzende Verbindung $\mathrm{C}_{17} \mathrm{H}_{16} \mathrm{O}$ läßt sich weder mit Essigsäureanhydrid noch mit einer Mischung von Essigsäureanhydrid und Natriumacetat acetylieren. Nach dem Erhitzen und Abkühlen der Lösung in Essigsäureanhydrid krystallisiert die Verbindung unverändert in großen, harten Krystallen aus. Mit Phenylhydrazin in warmer Eisessiglösung entstand ein krystallinisches Reaktionsprodukt, welches wegen seiner Zersetzlichkeit nicht näher untersucht werden konnte. Durch Reduktion mit Jodwasserstoffi und Phosphor geht die Verbindung $\mathrm{C}_{17} \mathrm{H}_{16} \mathrm{O}$ in Diphenylcyclopentan über. 